\title{
The Lowering of Ionization and Dissociation Energies of Molecules and Molecular Ions in Dense Hydrogen Plasma
}

\author{
S.I.Anisimov and Yu.V.Petrov \\ L.D.Landau Institute for Theoretical Physics \\ 142432 Chernogolovka, Moscow Region, Russia
}

\begin{abstract}
The influence of the screening of Coulomb interactions in plasma on the dissociation energy of molecules is considered. For the hydrogen molecule and its molecular ion the dissociation energy doesn't essentially depend upon the screening length whereas the lowering of their ionization potentials exeeds $30 \%$. This tendency makes more possible the ionization of hydrogen from the molecule through the molecular ion state.

[plasma, screening, ionization potentials, dissociation energy, hydrogen]
\end{abstract}

\section{Introduction}

When atoms are embedded into the plasma environment, the screening of the Coulomb interactions leads to the lowering of their ionization potentials as compared with the case of the isolated atoms $[1,2]$. Similar question arises concerning the influence of the plasma state onto the ionization and dissociation of molecules, particularly, when the molecular substances like the diatomic molecular hydrogen undergo the shock wave compression $[3,4]$. We've considered $H_{2}$-molecules in plasma with screened Coulomb interactions, so that the molecular Hamiltonian in the adiabatic approximation (with the use of atomic units) is:

$$
H=\sum_{i=1}^{2}\left(T_{i}-\sum_{p=a, b} U\left(r_{i p}\right)\right)+U\left(r_{12}\right)+U(R) .
$$

Here $T_{i}=-\frac{1}{2} \triangle_{i}$ is the kinetic energy of $i$-th electron, $U\left(r_{i p}\right)$ is the interaction energy between the electron spaced at $\mathbf{r}_{i}$ and the nucleus $p$, spaced at $\mathbf{R}_{p}(p=a, b)$; $r_{i p}=\left|\mathbf{r}_{i}-\mathbf{R}_{p}\right|$. Interelectronic and internuclear distances are: $r_{12}=\left|\mathbf{r}_{1}-\mathbf{r}_{2}\right|, R=\left|\mathbf{R}_{a}-\mathbf{R}_{b}\right|$ so that last two terms in (1) describe electron-electron and nuclearnuclear interactions. We take $U(r)$ in the form of the screened Coulomb (Debye-Hückel) interaction:

$$
U(r)=\frac{\exp (-r / \lambda)}{r} .
$$

\section{Hydrogen molecules and the screening}

We find the ground state energy of the molecule with the Hamiltonian (1) by the variational method using the trial function in the form of the linear combination of covalent $\Psi_{1}$ and ionic $\Psi_{2}$ and $\Psi_{3}$ valence bond basic functions:

$$
\Psi_{1}=(a \bar{b})-(b \bar{a}),
$$

$$
\begin{aligned}
& \Psi_{2}=(a \bar{a}), \\
& \Psi_{3}=(b \bar{b}) .
\end{aligned}
$$

Here

$$
(a \bar{b})=\left|\begin{array}{ll}
a(1) \alpha(1) & a(2) \alpha(2) \\
b(1) \beta(1) & b(2) \beta(2)
\end{array}\right|
$$

and $a(1)$ is the atomic orbital of the electron 1 , centered on the nucleus $a, \alpha=\left(\begin{array}{l}1 \\ 0\end{array}\right), \beta=\left(\begin{array}{l}0 \\ 1\end{array}\right)$ are different spin states of electron. Slater-type $1 s$-orbitals are approximated by the linear combinations of six Gauss-type $1 s$-orbitals

$$
a(1)=\sum_{i=1}^{6} c_{i} u_{i}\left(r_{1 a}\right)
$$

Here Gauss-type orbitals

$$
u_{i}(r)=\left(\frac{2 \alpha_{i} \xi^{2}}{\pi}\right)^{3 / 4} \exp \left(-\alpha_{i} \xi^{2} r^{2}\right)
$$

contain the variational parameter $\xi$ and the coefficients $c_{i}, \alpha_{i}$ are taken from [5]. Basic one- and two- electron integrals in gaussian functions arising when solving the secular problem with functions (3)-(5):

a) overlap integra!

$$
\left\langle a_{g}^{i} \mid b_{g}^{j}\right\rangle=\int u_{i}\left(\mathbf{r}-\mathbf{R}_{a}\right) u_{j}\left(\mathbf{r}-\mathbf{R}_{b}\right) d \mathbf{r},
$$

b) kinetic energy matrix element

$$
\left\langle a_{g}^{i}|t| b_{g}^{j}\right\rangle=\int u_{i}\left(\mathbf{r}-\mathbf{R}_{a}\right)\left(-\frac{1}{2} \Delta\right) u_{j}\left(\mathbf{r}-\mathbf{R}_{b}\right) d \mathbf{r},
$$

c) one-electron electrostatic matrix elements

$$
\begin{aligned}
& \left\langle a_{g}^{i}\left|U_{b}\right| a_{g}^{j}\right\rangle=\int u_{i}\left(\mathbf{r}-\mathbf{R}_{a}\right) U\left(\mathbf{r}-\mathbf{R}_{b}\right) u_{j}\left(\mathbf{r}-\mathbf{R}_{a}\right) d \mathbf{r}, \\
& \left\langle a_{g}^{i}\left|U_{a}\right| b_{g}^{j}\right\rangle=\int u_{i}\left(\mathbf{r}-\mathbf{R}_{a}\right) U\left(\mathbf{r}-\mathbf{R}_{a}\right) u_{j}\left(\mathbf{r}-\mathbf{R}_{b}\right) d \mathbf{r},
\end{aligned}
$$


d) two-electron electrostatic elements

$$
\begin{aligned}
& \left\langle a_{g}^{i} a_{g}^{j} \mid b_{g}^{k} b_{g}^{l}\right\rangle \\
& \quad=\int u_{i}\left(r_{1 a}\right) u_{j}\left(r_{1 a}\right) U\left(r_{12}\right) u_{k}\left(r_{2 b}\right) u_{l}\left(r_{2 b}\right) d \mathbf{r}_{1} d \mathbf{r}_{2} \\
& \left\langle a_{g}^{i} a_{g}^{j} \mid a_{g}^{k} b_{g}^{l}\right\rangle \\
& \quad=\int u_{i}\left(r_{1 a}\right) u_{j}\left(r_{1 a}\right) U\left(r_{12}\right) u_{k}\left(r_{2 a}\right) u_{l}\left(r_{2 b}\right) d \mathbf{r}_{1} d \mathbf{r}_{2} \\
& \left\langle a_{g}^{i} b_{g}^{j} \mid a_{g}^{k} b_{g}^{l}\right\rangle \\
& \quad=\int u_{i}\left(r_{1 a}\right) u_{j}\left(r_{1 b}\right) U\left(r_{12}\right) u_{k}\left(r_{2 a}\right) u_{l}\left(r_{2 b}\right) d \mathbf{r}_{1} d \mathbf{r}_{2}
\end{aligned}
$$

can be expressed in the analitical form.

\section{Results and discussion}

We've calculated the energies of $\mathrm{H}_{2}$-molecule and $\mathrm{H}_{2}^{+}$-molecular ion at different values of the screening length $\lambda$ of the interaction in plasma at fixed interatomic distances $R$ in the adiabatic approximation. The energy $E(R, \lambda)$ of the hydrogen molecule is shown in Fig.1.

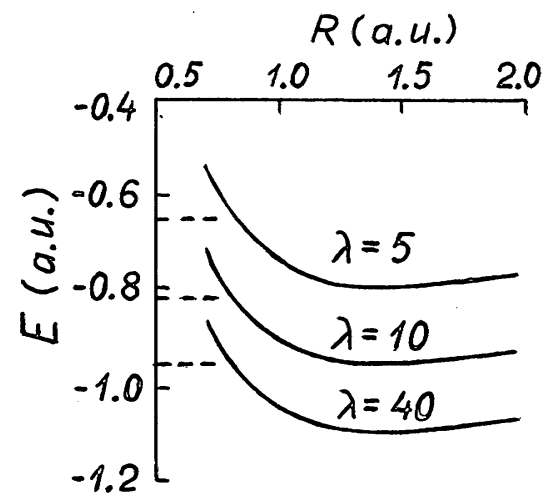

Fig.1. The energy of the $\mathrm{H}_{2}$-molecule as a function of the distance $R$ between the atoms at different values of the screening length of interaction $\lambda$. Dashed lines stand for twice the energy of the hydrogen atom at corresponding values of $\lambda$.

Several parameters of the curves presented in Fig.1, such as the equilibrium distance $R_{0}$ between the atoms in the molecule, and the distance $R_{*}$, for which the interaction energy between two atoms becomes equal to zero, are shown in the Table 1 for various values of the screening length $\lambda$. The third quantity, dissociation energy $D$, in Table 1 is obtained under the assumption that the energy of the interatomic interaction $V(R)$ can be described in terms of the Morse potential:

$$
V(R)=E(R, \lambda)-E(\infty, \lambda)=\epsilon\left(e^{-2 \beta x}-2 e^{-\beta x}\right) .
$$

Here $\epsilon$ is the depth of the potential well $\left(\epsilon=-V\left(R_{0}\right)\right)$ and $x=\left(R-R_{0}\right) / R_{0}$. Then the dissociation energy of $\mathrm{H}_{2}$-molecule with the atoms of the mass $M$ is equal to:

$$
D=\epsilon-\frac{\ln 2}{R_{0}-R_{*}} \sqrt{\frac{\epsilon}{M}}
$$

Table 1. The equilibrium interatomic distance $R_{0}$, the interatomic distance $R_{*}$ at which the interaction energy between two hydrogen atoms is equal to zero and the dissociation energy $D$ of the hydrogen molecules (all quantities are in the atomic units).

\begin{tabular}{|c|c|c|c|}
\hline$\lambda$ & $R_{0}$ & $R_{*}$ & $D$ \\
\hline 5 & 1.45561 & 0.83089 & 0.13340 \\
\hline 10 & 1.43673 & 0.82086 & 0.13666 \\
\hline 20 & 1.43367 & 0.81809 & 0.13760 \\
\hline 40 & 1.43057 & 0.81737 & 0.13782 \\
\hline 80 & 1.43152 & 0.81715 & 0.13791 \\
\hline
\end{tabular}

Whereas the total energy of the molecule is essentially increase with $\lambda$ decrease, it is accompanied at the same time by the increase of the ground state of the single atom, so that the difference between the energy of the molecule at the equilibrium distance and twice the atomic energy doesn't practically change when $\lambda$ decreases from the infinte value down to the value of $\lambda=5$. The equilibrium interatomic distance suffers only the small enhancement within this range of the screening length and the resulting dissociation energy remains essentially constant.

The total energy of $\mathrm{H}_{2}^{+}$-ion as the function of the internuclear distance $R$ and $\lambda$ is plotted in Fig.2.

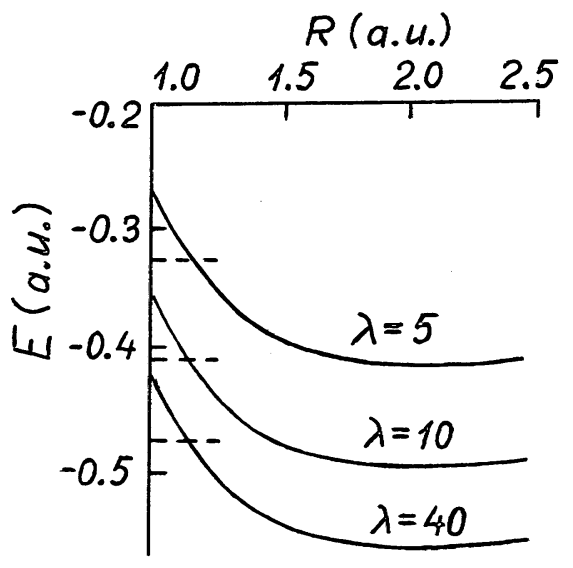

Fig.2.The energy of $\mathrm{H}_{2}^{+}$- ion vs the distance $R$ between the nuclei in it at several values of the screening length. Dashed lines correspond to the the single atom energy. 
As in the case of the neutral hydrogen molecule, interaction parameters in the hydrogen molecular ion, such as the equilibrium distance between the nuclei and the distance with zero interaction energy increases only slightly at the range of the screening length under consideration (see Table 2).

Table 2. The hydrogen molecular ion $H_{2}^{+}$length $R_{0}$, the distance $R_{*}$ between its nuclei at zero interaction energy and the dissociation energy $D$ of $H_{2}^{+}$(in the atomic units) in dependence on the screening length $\lambda$.

\begin{tabular}{|c|c|c|c|}
\hline$\lambda$ & $R_{0}$ & $R_{*}$ & $D$ \\
\hline 5 & 2.02305 & 1.15250 & 0.080574 \\
\hline 10 & 2.00652 & 1.14720 & 0.080990 \\
\hline 20 & 2.00454 & 1.14586 & 0.081040 \\
\hline 40 & 2.00362 & 1.14550 & 0.081039 \\
\hline 80 & 2.00327 & 1.14540 & 0.081037 \\
\hline
\end{tabular}

Again the growth of the single atom ground state energy (the lowering of the atomic ionization potential) which accompanies the increase of the total energy of the $H_{2}^{+}$-ion when $\lambda$ decreases from large to small values leads to the practically independent on $\lambda$ behaviour of the dissociation energy of the hydrogen molecular ion. The lowering of the ionization potential of the hydrogen atom in the same approximation as a function of the screening length of interaction $\lambda$ is plotted in Fig.3.

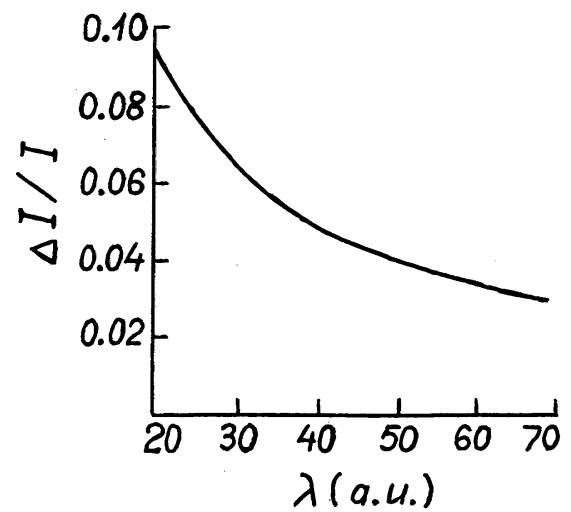

Fig.3. The relative lowering of the ionization potential of the hydrogen atom in dependence on the screening length $\lambda$.

When the total energies of the hydrogen molecule $E\left(H_{2}\right)$ and its ion $E\left(H_{2}^{+}\right)$are known, the ionization potentials $I_{1}$ and $I_{2}$ of the molecule can be calculated as
$I_{1}=E\left(H_{2}^{+}\right)-E\left(H_{2}\right)$ and $I_{2}=-E\left(H_{2}^{+}\right)$. For the finite values of $\lambda$ ionization potentials $I_{1}(\lambda)$ and $I_{2}(\lambda)$ differ from their values at very large screening length $I_{1}(\infty)$ and $I_{2}(\infty)$ when the screening can be ignored at all. The relative lowering of the ionization potentials of the hydrogen molecule $\Delta I / I=(I(\infty)-I(\lambda)) / I(\infty)$ are shown in Fig.4.

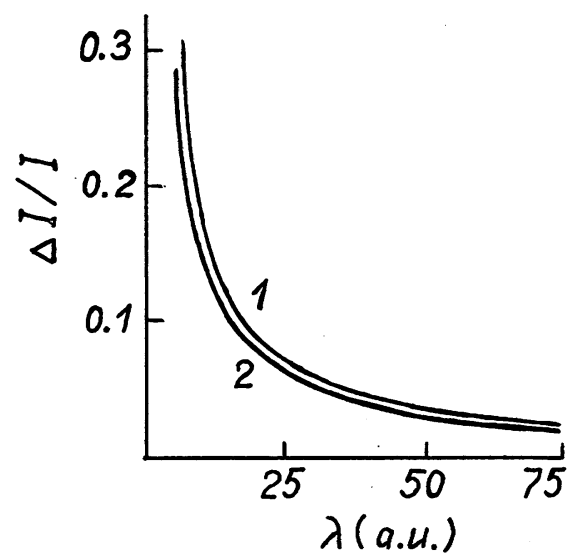

Fig.4. The relative lowering of the first (1) and second (2) ionization potentials of the hydrogen molecule in dependence on the screening length $\lambda$.

Our calculations with hydrogen molecules show that the decrease of the screening length resulting in the essential lowering of the ionization potentials of molecules doesn't practically change their dissociation energies. This tendency magnifies the possibility of the ionization of the hydrogen molecules in the molecular phase through the creation of the hydrogen molecular ion.

\section{Acknowledgments}

This work was supported in part by the Grants of the Russian Fund for Fundamental Research RFFR-95-0204535a and RFFR-95-02-06381a

\section{References}

[1] K.Kilimann and W.Ebeling, Phys.Lett., A61,393 (1977).

[2] W.Ebeling et al., Thermophysical Propertis of Hot Dense Plasmas, B.G.Teubner Verlagsgesellschaft, Stuttgart-Leipzig, 1991.

[3] W.J.Nellis et al., Phys.Rev.Lett., 68,2937(1992). [4] S.T.Weir, A.C.Mitchell and W.J.Nellis, Phys. Rev. Lett., 76,1860(1996).

[5] G.Page and O.Ludwig, J.Chem.Phys., 56, 5626 (1972). 